\title{
Discovery and Verification of Neighbor Positions in Mobile Ad Hoc Networks
}

\author{
Marco Fiore, Member, IEEE, Claudio Casetti, Member, IEEE, Carla-Fabiana Chiasserini, Senior Member, IEEE, \\ Panagiotis Papadimitratos, Member, IEEE
}

\begin{abstract}
A growing number of ad hoc networking protocols and location-aware services require that mobile nodes learn the position of their neighbors. However, such a process can be easily abused or disrupted by adversarial nodes. In absence of a-priori trusted nodes, the discovery and verification of neighbor positions presents challenges that have been scarcely investigated in the literature. In this paper, we address this open issue by proposing a fully-distributed cooperative solution that is robust against independent and colluding adversaries, and can be impaired only by an overwhelming presence of adversaries. Results show that our protocol can thwart more than $99 \%$ of the attacks under the best possible conditions for the adversaries, with minimal false positive rates.
\end{abstract}

Index Terms-Neighbor position verification, mobile ad hoc networks, vehicular networks.

\section{INTRODUCTION}

Location awareness has become an asset in mobile systems, where a wide range of protocols and applications require knowledge of the position of the participating nodes. Geographic routing in spontaneous networks, data gathering in sensor networks, movement coordination among autonomous robotic nodes, location-specific services for handheld devices, and danger warning or traffic monitoring in vehicular networks are all examples of services that build on the availability of neighbor position information.

The correctness of node locations is therefore an allimportant issue in mobile networks, and it becomes particularly challenging in the presence of adversaries aiming at harming the system. In these cases, we need solutions that let nodes (1) correctly establish their location in spite of attacks feeding false location information, and (2) verify the positions of their neighbors, so as to detect adversarial nodes announcing false locations.

In this paper, we focus on the latter aspect, hereinafter referred to as neighbor position verification (NPV for short). Specifically, we deal with a mobile ad hoc network, where a pervasive infrastructure is not present, and the location data must be obtained through node-to-node communication. Such a scenario is of particular interest since it leaves the door open for adversarial nodes to misuse or disrupt the locationbased services. For example, by advertising forged positions, adversaries could bias geographic routing or data gathering

M. Fiore is with Université de Lyon, INSA Lyon, INRIA, CITI, F-69621, France, e-mail: marco.fiore@insa-lyon.fr.

C. Casetti and C.-F. Chiasserini are with the Dipartimento di Elettronica, Politecnico di Torino, Torino, Italy, email: lastname@ polito.it.

P. Papadimitratos is with the School of Electrical Engineering at KTH, Stockholm, Sweden, email: papadim@kth.se. processes, attracting network traffic and then eavesdropping or discarding it. Similarly, counterfeit positions could grant adversaries unauthorized access to location-dependent services, let vehicles forfeit road tolls, disrupt vehicular traffic or endanger passengers and drivers.

In this context, the challenge is to perform, in absence of trusted nodes, a fully-distributed, lightweight NPV procedure that enables each node to acquire the locations advertised by its neighbors, and assess their truthfulness. We therefore propose an NPV protocol that has the following features:

- It is designed for spontaneous ad hoc environments, and, as such, it does not rely on the presence of a trusted infrastructure or of a-priori trustworthy nodes;

- It leverages cooperation but allows a node to perform all verification procedures autonomously. This approach has no need for lengthy interactions, e.g., to reach a consensus among multiple nodes, making our scheme suitable for both low- and high-mobility environments;

- It is reactive, meaning that it can be executed by any node, at any point in time, without prior knowledge of the neighborhood;

- It is robust against independent and colluding adversaries;

- It is lightweight, as it generates low overhead traffic.

Additionally, our NPV scheme is compatible with state-of-theart security architectures, including the ones that have been proposed for vehicular networks [1], [2], which represent a likely deployment environment for NPV.

The rest of the paper is organized as follows. In Sec. II we review previous works, highlighting the novelty of our solution. In Sec. III we describe the system model, while the communication protocol, the objectives of the verification procedure and our main results are outlined in Sec. IV. The details of the NPV protocol and of verification tests are then presented in Sec. V, and the resilience of our solution to different attacks is analyzed in Sec. VI. Finally, we provide a performance evaluation of the protocol in a vehicular scenario in Sec. VII, and draw conclusions in Sec. VIII.

\section{RELATED WORK}

Although the literature carries a multitude of ad hoc security protocols addressing a number of problems related to NPV, there are no lightweight, robust solutions to NPV that can operate autonomously in an open, ephemeral environment, without relying on trusted nodes. Below, we list relevant works and highlight the novelty of our contribution. For clarity of presentation, we first review solutions to some NPV-related 
problems, such as secure positioning and secure discovery, and then we discuss solutions specifically addressing NPV.

Securely determining own location. In mobile environments, self-localization is mainly achieved through Global Navigation Satellite Systems, e.g., GPS, whose security can be provided by cryptographic and non-cryptographic defense mechanisms [3]. Alternatively, terrestrial special-purpose infrastructure could be used [4], [5], along with techniques to deal with non-honest beacons [6]. We remark that this problem is orthogonal to the problem of NPV. In the rest of this paper, we will assume that devices employ one of the techniques above to securely determine their own position and time reference.

Secure neighbor discovery (SND) deals with the identification of nodes with which a communication link can be established or that are within a given distance [7]. SND is only a step towards the solution we are after: simply put, an adversarial node could be securely discovered as neighbor and be indeed a neighbor (within some SND range), but it could still cheat about its position within the same range. In other words, SND is a subset of the NPV problem, since it lets a node assess whether another node is an actual neighbor but it does not verify the location it claims to be at. SND is most often employed to counter wormhole attacks [8]-[10]; practical solutions to the SND problem have been proposed in [11], while properties of SND protocols with proven secure solutions can be found in [12], [13].

Neighbor position verification was studied in the context of ad-hoc and sensor networks; however, existing NPV schemes often rely on fixed [14], [15] or mobile [16] trustworthy nodes, which are assumed to be always available for the verification of the positions announced by third parties. In ad hoc environments, however, the pervasive presence of either infrastructure or neighbor nodes that can be aprioristically trusted is quite unrealistic. Thus, we devise a protocol that is autonomous and does not require trustworthy neighbors.

In [17], an NPV protocol is proposed that first lets nodes calculate distances to all neighbors, and then commends that all triplets of nodes encircling a pair of other nodes act as verifiers of the pair's positions. This scheme does not rely on trustworthy nodes, but it is designed for static sensor networks, and requires lengthy multi-round computations involving several nodes that seek consensus on a common neighbor verification. Furthermore, the resilience of the protocol in [17] to colluding attackers has not been demonstrated. The scheme in [18] suits static sensor networks too, and it requires several nodes to exchange information on the signal emitted by the node whose location has to be verified. Moreover, it aims at assessing not the position but whether the node is within a given region or not. Our NPV solution, instead, allows any node to validate the position of all of its neighbors through a fast, one-time message exchange, which makes it suitable to both static and mobile environments. Additionally, we show that our NPV scheme is robust against several different colluding attacks. Similar differences can be found between our work and [19].

In [20], the authors propose an NPV protocol that allows nodes to validate the position of their neighbors through local observations only. This is performed by checking whether subsequent positions announced by one neighbor draw a movement over time that is physically possible. The approach in [20] forces a node to collect several data on its neighbor movements before a decision can be taken, making the solution unfit to situations where the location information is to be obtained and verified in a short time span. Moreover, an adversary can fool the protocol by simply announcing false positions that follow a realistic mobility pattern. Conversely, by exploiting cooperation among nodes, our NPV protocol is (i) reactive, as it can be executed at any instant by any node, returning a result in a short time span, and (ii) robust to fake, yet realistic, mobility patterns announced by adversarial nodes over time.

The scheme in [21] exploits Time-of-Flight distance bounding and node cooperation to mitigate the problems of the previous solutions. However, the cooperation is limited to couples of neighbor nodes, which renders the protocol ineffective against colluding attackers.

To our knowledge, our protocol is the first to provide a fully distributed, lightweight solution to the NPV problem that does not require any infrastructure or a-priori trusted neighbors and is robust to several different attacks, including coordinated attacks by colluding adversaries. Also, unlike previous works, our solution is suitable for both low and high mobile environments and it only assumes RF communication. Indeed, nonRF communication, e.g., infra-red or ultra-sound, is unfeasible in mobile networks, where non-line-of-sight conditions are frequent and device-to-device distances can be in the order of tens or hundreds of meters. An early version of this work, sketching the NPV protocol and some of the verification tests to detect independent adversaries, can be found in [22].

\section{SYSTEM AND ADVERSARY MODEL}

We consider a mobile network and define as communication neighbors of a node all the other nodes that it can reach directly with its transmissions [7]. We assume that each node knows its own position with some maximum error $\epsilon_{p}$, and that it shares a common time reference with the other nodes: both requirements can be met by equipping communication nodes with GPS receivers ${ }^{1}$. In addition, nodes can perform Time of Flight (ToF)-based RF ranging with a maximum error equal to $\epsilon_{r}$. As discussed in [17], this is a reasonable assumption, although it requires modifications to off-the-shelf radio interfaces; also, promising techniques for precise ToFbased RF ranging have been developed [24].

We assume that node positions do not vary significantly during a protocol execution, since a complete message exchange takes no more than a few hundreds of milliseconds. The relative spatial movements of the nodes during such a period are taken into account through the tolerance value $\epsilon_{m}$.

Nodes carry a unique identity ${ }^{2}$ and can authenticate messages of other nodes through public key cryptography [27]. In particular, we assume that each node $X$ owns a private key,

\footnotetext{
${ }^{1}$ Small-footprint GPS receivers are commercially available, which achieve low synchronization and localization errors [23].

${ }^{2}$ This can be a permanent identifier or a temporary pseudonym, so as to ensure user privacy [25].
} 
$k_{X}$, and a public key, $K_{X}$, as well as a set of one-time use keys $\left\{k_{X}^{\prime}, K_{X}^{\prime}\right\}$, as proposed in emerging architectures for secure and privacy-enhancing communication [2], [25]. Node $X$ can encrypt and decrypt data with its keys and the public keys of other nodes; also, it can produce digital signatures $\left(\right.$ Sig $\left._{X}\right)$ with its private key. We assume that the binding between $X$ and $K_{X}$ can be validated by any node, as in state-of-the-art secure communication architectures [2], [26].

Nodes are correct if they comply with the NPV protocol, and adversarial if they deviate from it. As authentication essentially thwarts external adversaries, we focus on the more powerful internal ones, i.e., nodes that possess the cryptographic material to participate in the NPV and try to exploit it, by advertising arbitrarily erroneous own positions or inject misleading information. Internal adversaries cannot forge messages on behalf of other nodes whose keys they do not have. Thus, attacks against the cryptosystem are not considered, as correct implementation of cryptographic primitives makes them computationally infeasible.

We further classify adversaries into: knowledgeable, if at each time instant they know positions and (temporary) identities of all their communication neighbors, and unknowledgeable, otherwise; independent, if they act individually, and colluding, if they coordinate their actions.

\section{Cooperative NPV: An Overview}

We propose a fully-distributed cooperative scheme for NPV, which enables a node, hereinafter called the verifier, to discover and verify the position of its communication neighbors. For clarity, here we summarize the principles of the protocol as well as the gist of its resilience analysis. Detailed discussions of message format, verification tests and protocol resilience are provided in Sec. V and Sec. VI.

A verifier, $S$, can initiate the protocol at any time instant, by triggering the 4-step message exchange depicted in Fig. 1, within its 1-hop neighborhood. The aim of the message exchange is to let $S$ collect information it can use to compute distances between any pair of its communication neighbors. To that end, POLL and REPLY messages are first broadcasted by $S$ and its neighbors, respectively. These messages are anonymous and take advantage of the broadcast nature of the wireless medium, allowing nodes to record reciprocal timing information without disclosing their identities. Then, after a REVEAL broadcast by the verifier, nodes disclose to $S$, through secure and authenticated REPORT messages, their identities as well as the anonymous timing information they collected. The verifier $S$ uses such data to match timings and identities; then, it uses the timings to perform ToF-based ranging and compute distances between all pairs of communicating nodes in its neighborhood.

Once $S$ has derived such distances, it runs several position verification tests in order to classify each candidate neighbor as either:

1) Verified, i.e., a node the verifier deems to be at the claimed position;

2) Faulty, i.e., a node the verifier deems to have announced an incorrect position;

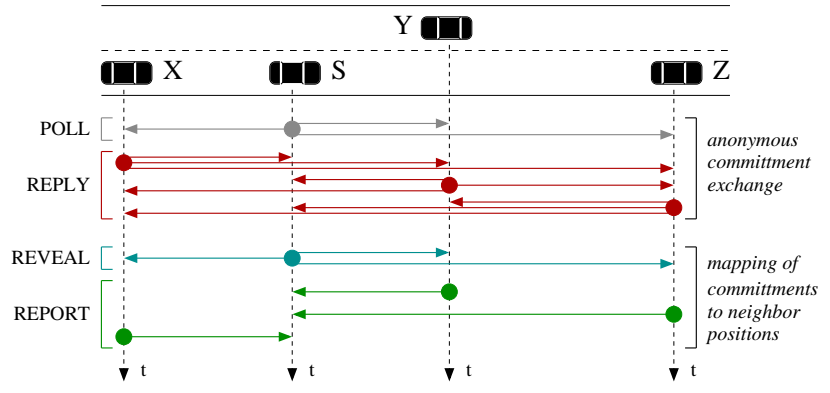

Fig. 1. Message exchange overview, during one instance of the NPV protocol.

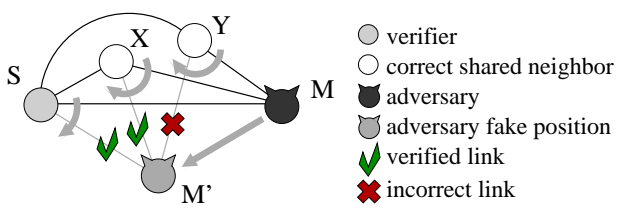

Fig. 2. Example of topological information stored by verifier $S$ at the end of the message exchange and effect of a fake position announcement by $M$.

3) Unverifiable, i.e., a node the verifier cannot prove to be either correct or faulty, due to insufficient information.

Clearly, the verification tests aim at avoiding false negatives (i.e., adversaries announcing fake positions that are deemed verified) and false positives (i.e., correct nodes whose positions are deemed faulty), as well as at minimizing the number of unverifiable nodes. We remark that our NPV scheme does not target the creation of a consistent "map" of neighborhood relations throughout an ephemeral network: rather, it allows the verifier to independently classify its neighbors.

The basic principle the verification tests build upon is best explained by means of the example in Fig. 2. There, $M$ is a malicious node announcing a false location $M^{\prime}$, so as to fraudulently gain some advantage over other nodes. The figure portrays the actual network topology with black edges, while the modified topology, induced by the fake position announced by $M$, is shown with gray edges. It is evident that the displacement of $M$ to $M^{\prime}$ causes its edges with the other nodes to rotate, which, in turn, forces edge lengths to change as well. The tests thus look for discrepancies in the node distance information to identify incorrect node positions.

A malicious node, knowing the protocol, can try to outsmart the tests in a number of different ways. Sec. VI contains a comprehensive discussion of the protocol resilience, covering conceivable attack strategies that adversarial nodes could adopt. Overall, our analysis proves that:

- An unknowledgeable adversary has no possibility of success against our NPV protocol;

- An independent knowledgeable adversary $M$ can move at most two links (with the verifier $S$ and with a shared neighbor $X$ ) without being detected: however, any additional link (e.g., with another shared neighbor $Y$ ) leads to inconsistencies between distances and positions that allow to identify the attacker: this is the situation depicted in Fig. 2. In a nutshell, independent adversaries, although knowledgeable, cannot harm the system;

- Colluding knowledgeable adversaries can announce tim- 
TABLE I

SUMMARY OF NOTATIONS

\begin{tabular}{ll}
\hline Notation & Description \\
\hline$k_{X}\left(K_{X}\right)$ & private (public) key of $X$ \\
$k_{X}^{\prime}\left(K_{X}^{\prime}\right)$ & private (public) one-time key of $X$ \\
$t_{X}\left(t_{X}^{\prime}\right)$ & actual (fake) transmission time of a message by $X$ \\
$t_{X Y}\left(t_{X Y}^{\prime}\right)$ & actual (fake) reception time at $Y$ of a message by $X$ \\
$p_{X}\left(p_{X}^{\prime}\right)$ & actual (fake) position of $X$ \\
$d_{X Y}$ & distance between $X$ and $Y$ \\
$\epsilon_{p}\left(\epsilon_{r}\right)$ & position (ranging) error \\
$\epsilon_{m}$ & tolerance to node movements during protocol execution \\
$R$ & node proximity range \\
$\mathbb{N}_{X}$ & current set of $X$ 's comm. neighbors \\
$T_{X}$ & random wait interval after PoLL reception at $X$ \\
$\rho_{X}$ & nonce sent by $X$ \\
$S i g_{X}$ & digital signature of $X$ \\
$C_{X}$ & certificate of $X$ \\
$\mathbb{C}_{X}$ & commitment of $X$ \\
$i_{X}$ & temporary identifier assigned by $S$ to $X$ \\
$\mathbb{V}_{X}$ & set of verified comm. neighbors of $X$ \\
$\mathbb{U}_{X}$ & set of unverifiable comm. neighbors of $X$ \\
$\mathbb{F}_{X}$ & set of faulty comm. neighbors of $X$ \\
$\mathbb{W}_{X}$ & set of conditionally verified comm. neighbors of $X$ \\
&
\end{tabular}

ing information that reciprocally validate their distances, and pose a more dangerous threat to the system. However, we prove that an overwhelming presence of colluders in the verifier neighborhood is required for an attack to be successful. Additionally, simulations in realistic scenarios prove the robustness of the NPV protocol even against large groups of colluding knowledgeable adversaries.

\section{NPV PROTOCOL}

We detail the message exchange between the verifier and its communication neighbors, followed by a description of the tests run by the verifier. Table I summarizes the notations used throughout the protocol description.

\section{A. Protocol message exchange}

The value $p_{X}$ is the current position of $X$, and $\mathbb{N}_{X}$ is the current set of its communication neighbors. We denote by $t_{X}$ the time at which a node $X$ starts a broadcast transmission and by $t_{X Y}$ the time at which a node $Y$ starts receiving it. Note that these time values refer to the actual instant at which the node starts transmitting/receiving the first bit of the message at the physical layer. To retrieve the exact transmission and reception time instants, avoiding the unpredictable latencies introduced by interrupts triggered at the drivers level, a solution such as that implemented in [28] is required ${ }^{3}$. Furthermore, the GPS receiver should be integrated in the 802.11 card; software defined radio solutions combining GPS and 802.11 capabilities are proposed, among others, in [29], [30].

Now, consider a verifier $S$ that initiates the NPV protocol. The message exchange procedure is outlined in Alg. 1 for $S$, and in Alg. 2 for any of $S$ 's communication neighbors.

POLL message. The verifier starts the protocol by broadcasting a POLL whose transmission time $t_{S}$ it stores locally (Alg. 1, lines 2-3). The POLL is anonymous, since (i) it does

\footnotetext{
${ }^{3}$ This leads to a timing precision of around $23 \mathrm{~ns}$, dictated by the $44 \mathrm{MHz}$ clock of standard $802.11 \mathrm{a} / \mathrm{b} / \mathrm{g}$ cards. As mentioned above, we account for these errors through the $\epsilon_{r}$ parameter.
}

not carry the identity of the verifier, (ii) it is transmitted employing a fresh, software-generated MAC address, and (iii) it contains a public key $K_{S}^{\prime}$ taken from $S$ 's pool of anonymous one-time use keys that do not allow neighbors to map the key onto a specific node. We stress that keeping the identity of the verifier hidden is important in order to make our NPV robust to attacks (see the protocol analysis in Sec. VI). Since a source address has to be included in the MAC-layer header of the message, a fresh, software-generated MAC address is needed; note that this is considered a part of emerging cooperative systems [2], [25]. Including a one-time key in the POLL also ensures that the message is fresh (i.e., the key acts as a nonce).

REPLY message. A communication neighbor $X \in \mathbb{N}_{S}$ that receives the POLL stores its reception time $t_{S X}$, and extracts a random wait interval $T_{X} \in\left[0, T_{\max }\right]$ (Alg.. 2, lines 2-4). After $T_{X}$ has elapsed, $X$ broadcasts an anonymous REPLY message using a fresh MAC address, and locally records its transmission time $t_{X}$ (Alg. 2, lines 5-9). For implementation feasibility, the physical layer transmission time cannot be stamped on the REPLY, but it is stored by $X$ for later use. The REPLY contains some information encrypted with $S$ 's public key $\left(K_{S}^{\prime}\right)$, specifically the POLL reception time and a nonce $\rho_{X}$ used to tie the REPLY to the next message sent by $X$ : we refer to these data as $X$ 's commitment, $\mathbb{C}_{X}$ (Alg. 2, line 7). The hash $h_{K_{S}^{\prime}}$, derived from the public key of the verifier, $K_{S}^{\prime}$, is also included to bind POLL and REPLY belonging to the same message exchange.

Upon reception of a REPLY from a neighbor $X$, the verifier $S$ stores the reception time $t_{X S}$ and the commitment $\mathbb{C}_{X}$ (Alg. 1, lines 4-5). When a different neighbor of $S$, e.g., $Y, Y \in \mathbb{N}_{S} \cap \mathbb{N}_{X}$, broadcasts a REPLY too, $X$ stores the reception time $t_{Y X}$ and the commitment $\mathbb{C}_{Y}$ (Alg. 2, lines 1011). Since REPLY messages are anonymous, a node records all commitments it receives without knowing their originators.

REVEAL message. After a time $T_{\max }+\Delta+T_{j i t t e r}$, the verifier broadcasts a REVEAL message using its real MAC address (Alg. 1, line 6). $\Delta$ accounts for the propagation and contention lag of REPLY messages scheduled at time $T_{\max }$, and $T_{\text {jitter }}$ is a random time added to thwart jamming efforts on this message. The REVEAL contains: (i) a map $m_{S}$, that associates each commitment $\mathbb{C}_{X}$ received by the verifier to a temporary identifier $i_{X}$ (Alg. 1, line 7); (ii) a proof that $S$ is the author of the original POLL, through the encrypted hash $E_{k_{S}^{\prime}}\left\{h_{K_{S}^{\prime}}\right\}$; (iii) the verifier identity, i.e., its certified public key and signature (Alg. 1, line 8). Note that using certified keys curtails continuous attempts at running the protocol by an adversary who aims at learning neighbor positions (i.e., at becoming knowledgeable) or at launching a clogging attack (see Sec. VI-D).

REPORT message. Once the REVEAL message is broadcast and the identity of the verifier is known, each neighbor $X$ that previously received $S$ 's POLL unicasts to $S$ an encrypted, signed REPORT message. The REPORT carries $X$ 's position, the transmission time of $X$ 's REPLY, and the list of pairs of reception times and temporary identifiers referring to the REPLY broadcasts $X$ received (Fig. 2, lines 12-14). The identifiers are obtained from the map $m_{S}$ included in the REVEAL message. Also, $X$ discloses its own identity by including in the 

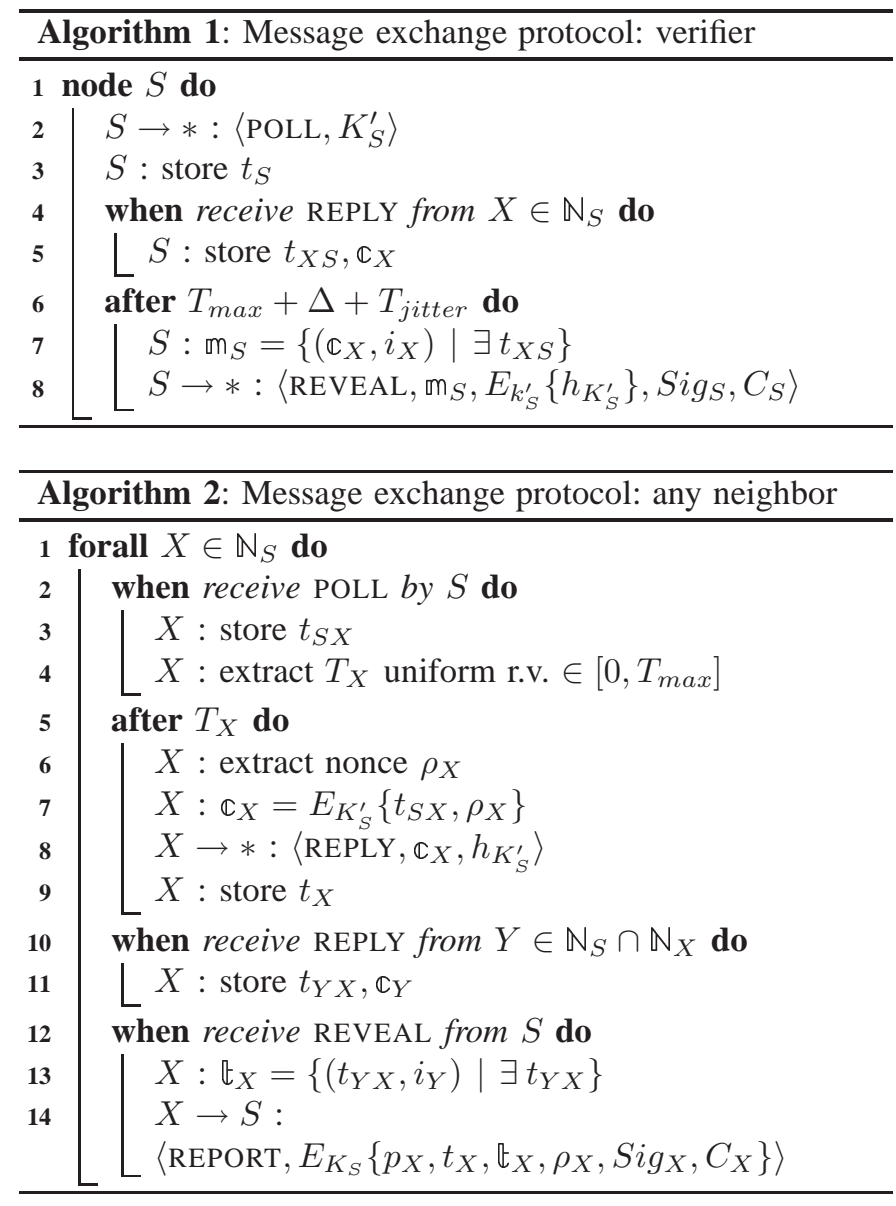

message its digital signature and certified public key; through the nonce $\rho_{X}$, it correlates the REPORT to its previously issued REPLY. We remark that all sensitive data are encrypted using $S$ 's public key, $K_{S}$, so that eavesdropping on the wireless channel is not possible. At the end of the message exchange, only the verifier knows all positions and timing information. If needed, certified keys in REPORT messages allow the matching of such data and node identities (temporary or long-term, with the help of an authority if needed [2].)

\section{B. Position verification}

Once the message exchange is concluded, $S$ can decrypt the received data and acquire the position of all neighbors that participated in the protocol, i.e., $\left\{p_{X}, \forall X \in \mathbb{N}_{S}\right\}$. The verifier $S$ also knows the transmission time $t_{S}$ of its POLL and learns that of all subsequent REPLY messages, i.e., $\left\{t_{X}, \forall X \in \mathbb{N}_{S}\right\}$, as well as the corresponding reception times recorded by the recipients of such broadcasts, i.e., $\left\{t_{X Y}, \forall X, Y \in \mathbb{N}_{S} \cup\{S\}\right\}$. Applying a ToF-based technique, $S$ thus computes its distance from each communication neighbor, as well as the distances between all neighbor pairs sharing a link. More precisely, by denoting with $c$ the speed of light, the verifier computes, for any communicating pair $(X, Y)$ with $X, Y \in \mathbb{N}_{S} \cup\{S\}$, two distances: $d_{X Y}=\left(t_{X Y}-t_{X}\right) \cdot c$, from the timing information related to the broadcast message sent by $X$, and $d_{Y X}=\left(t_{Y X}-t_{Y}\right) \cdot c$, from the information related to the broadcast message by $Y$.

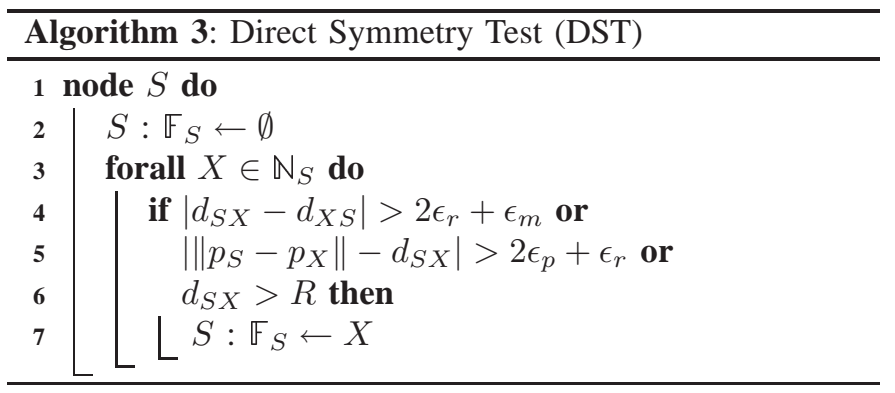

Once such distances have been computed, $S$ can run the following three verification tests to fill the sets $\mathbb{F}_{S}, \mathbb{V}_{S}$ and $\mathbb{U}_{S}$ with, respectively, faulty, verified and unverifiable nodes.

1) The Direct Symmetry Test (DST) is the first verification performed by $S$ and is detailed in Alg. 3. There, $|\cdot|$ denotes the absolute value operator and $\left\|p_{X}-p_{Y}\right\|$ the Euclidean distance between locations $p_{X}$ and $p_{Y}$. In the DST, $S$ verifies the direct links with its communication neighbors. To this end, it checks whether reciprocal ToF-derived distances are consistent (i) with each other, (ii) with the position advertised by the neighbor, and (iii) with a proximity range $R$. The latter corresponds to the maximum nominal transmission range, and upperbounds the distance at which two nodes can communicate. More specifically, the first check verifies that the distances $d_{S X}$ and $d_{X S}$, obtained from ranging, do not differ by more than twice the ranging error plus a tolerance value $\epsilon_{m}$ (Alg. 3, line 4), accounting for node spatial movements during the protocol execution. The second check verifies that the position advertised by the neighbor is consistent with such distances, within an error margin of $2 \epsilon_{p}+\epsilon_{r}$ (Alg. 3, line 5). Although trivial, this check is fundamental since it correlates positions to computed distances: without it, an attacker could fool the verifier by simply advertising an arbitrary position along with correct broadcast transmission and reception timings. Finally, as a sanity check, $S$ verifies that $d_{S X}$ is not larger than $R$ (Alg. 3, line 6). The verifier tags a neighbor as faulty if a mismatch is found in any of these checks ${ }^{4}$, since this implies an inconsistency between the position $p_{X}$ and the timings announced by the neighbor $\left(t_{S X}, t_{X}\right)$ or recorded by the verifier $\left(t_{X S}, t_{S}\right)$.

2) The Cross-Symmetry Test (CST), in Alg. 4, implements cross-verifications, i.e., it checks on the information mutually gathered by each pair of communication neighbors. The CST ignores nodes already declared as faulty by the DST (Alg. 4, line 5) and only considers nodes that proved to be communication neighbors between each other, i.e., for which ToF-derived mutual distances are available (Alg. 4, line 6). However, pairs of neighbors declaring collinear positions with respect to $S$ are not taken into account (Alg. 4, line 7, where line $\left(p_{X}, p_{Y}\right)$ is the line passing by points $p_{X}$ and $p_{Y}$ ). As shown in the next section, this choice makes our NPV robust to attacks in particular situations. For all other pairs $(X, Y)$, the CST verifies the symmetry of the reciprocal distances (Alg. 4, line 9), their consistency with the positions declared by the nodes (Alg. 4, line 10), and with the proximity range (Alg. 4, line

\footnotetext{
${ }^{4}$ The latter two checks are performed on both $d_{S X}$ and $d_{X S}$, however in Alg. 3 they are done on $d_{S X}$ only, for clarity of presentation.
} 


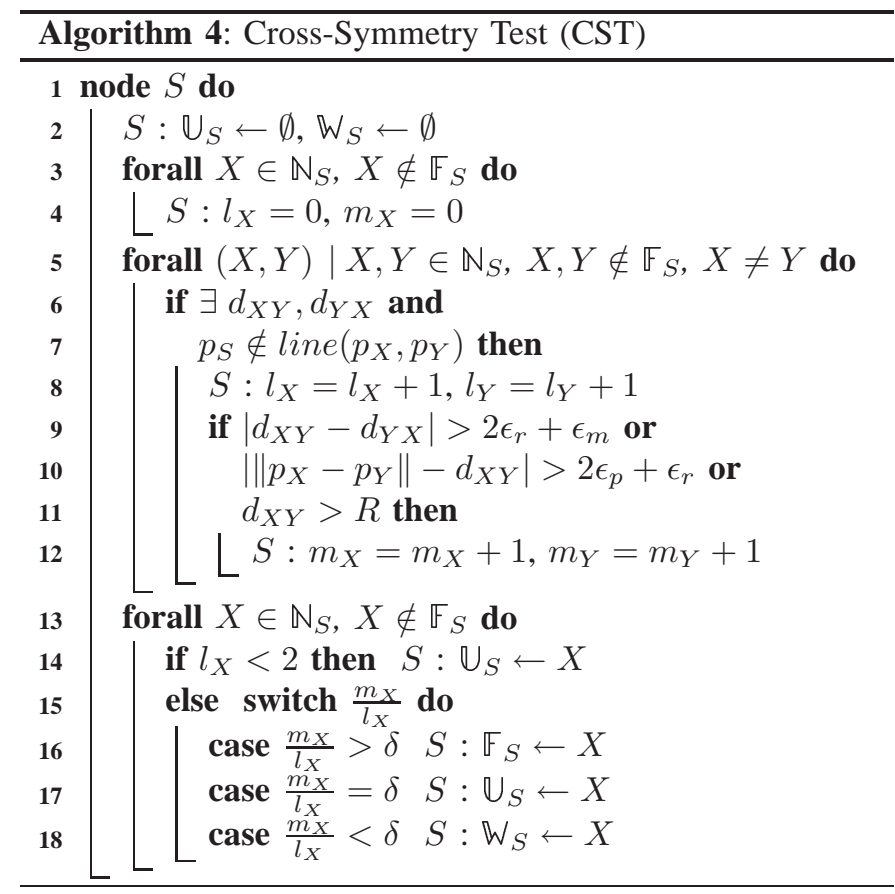

11). For each neighbor $X, S$ maintains a link counter $l_{X}$ and a mismatch counter $m_{X}$. The former is incremented at every new cross-check on $X$, and records the number of links between $X$ and other neighbors of $S$ (Alg. 4, line 8). The latter is incremented every time at least one of the cross-checks on distance and position fails (Alg. 4, line 12), and identifies the potential for $X$ being faulty.

Once all neighbor pairs have been processed, a node $X$ is added to the unverifiable set $\mathbb{U}_{S}$ if it shares less than two non-collinear neighbors with $S$ (Alg. 4, line 14). Indeed, in this case, the information available on the node is considered to be insufficient to tag the node as verified or faulty (see Sec. VI for details). Otherwise, if $S$ and $X$ have two or more non-collinear common neighbors, $X$ is declared as faulty, unverifiable, or conditionally verified, depending on the percentage of mismatches in the cross-checks it was involved in (Alg. 4, lines 15-18). Specifically, $X$ is added to $\mathbb{F}_{S}$ or $\mathbb{U}_{S}$, depending on whether the ratio of the number of mismatches to the number of checks is greater or equal to a threshold $\delta$. If such a ratio is less than $\delta, X$ is added to a temporary set $W_{S}$ for conditionally verified nodes.

We point out that the lower the $\delta$, the higher the probability of false positives, while the higher the $\delta$, the higher the probability of false negatives. In the following, we set $\delta=0.5$ so that the verifier makes a decision on the correctness of a node by relying on the opinion of the majority of shared (non-collinear) communication neighbors. As shown later, this choice makes our NPV highly resilient to attacks, unless the presence of adversaries becomes overwhelming.

3) The Multilateration Test (MLT), in Alg. 5, ignores nodes already tagged as faulty or unverifiable and looks for suspect neighbors in $\mathbb{W}_{S}$. For each neighbor $X$ that did not notify about a link reported by another node $Y$, with $X, Y \in \mathbb{W}_{S}$, a curve $L_{X}(S, Y)$ is computed and added to the set $\mathbb{L}_{X}$ (Alg. 5, lines 5-7). Such a curve is the locus of

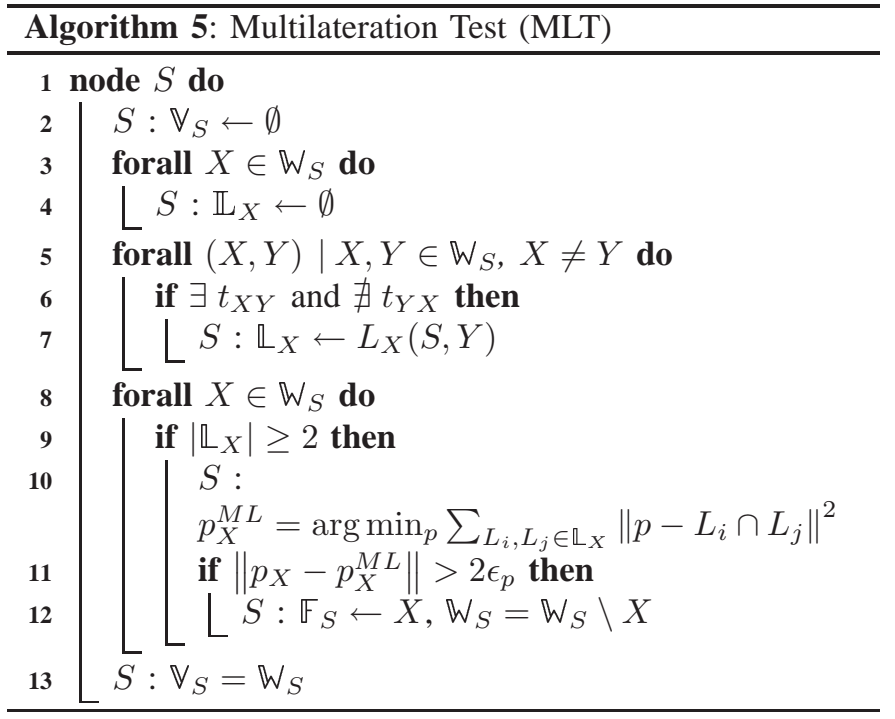

points that can generate a transmission whose Time Difference of Arrival (TDoA) at $S$ and $Y$ matches that measured by the two nodes, i.e., $\left|t_{X S}-t_{X Y}\right|$. It is easy to verify that such a curve is a hyperbola, with foci in $p_{S}$ and $p_{Y}$, and passing through the actual position of $X$.

Once all couples of nodes in $\mathbb{W}_{S}$ have been checked, each node $X$ for which two or more unnotified links, hence two or more hyperbolas in $\mathbb{L}_{X}$, exist is considered as suspect (Alg. 5, line 9). In such a case, $S$ exploits the hyperbolae in $\mathbb{L}_{X}$ to multilaterate $X$ 's position, referred to as $p_{X}^{M L}$, similarly to what is done in [17] (Alg. 5, line 10). Note that $\mathbb{L}_{X}$ must include at least two hyperbolae for $S$ to be able to compute $p_{X}^{M L}$, and this implies the presence of at least two shared neighbors between $S$ and $X . p_{X}^{M L}$ is then compared with the position advertised by $X, p_{X}$ (Alg. 5, line 11). If the difference exceeds an error margin $2 \epsilon_{p}, X$ is moved to the faulty set $\mathbb{F}_{S}$. At the end of the test, all nodes still in $\mathbb{W}_{S}$ are tagged as verified and moved to $\mathbb{V}_{S}$ (Alg. 5, lines 12-13).

\section{RESILIENCE ANALYSIS}

We analyze the robustness of our scheme against different types of internal adversaries. We classify the conceivable attacks into two classes, depending on the goal of the adversaries:

- Attacks where the adversaries aim at letting the verifier validate their own fake position;

- Attacks where the adversaries aim at disrupting the verification of correct node positions.

We focus on attacks of the first category in Sec. VI-A and Sec. VI-B, where we discuss the case of independent and colluding adversaries, respectively. In case of attacks of this first type, adversaries can tamper with the timing information in the REPLYS and REPORTs they generate, so that these confirm their false advertised locations. By considering the geometrical properties of the ToF-based ranging, we analyze the entire space of attacks against NPV. The effects of combinations of attacks of the first type is then investigated in our performance evaluation. 
Attacks of the second category are analyzed in Sec. VI-C, where adversaries try to induce the verifier to tag a correct neighbor as faulty or unverifiable.

Finally, in Sec. VI-D we discuss the robustness of our NPV scheme to generic attacks that are not specific to NPV. It is worth remarking that the NPV verification tests disregard nodes for which incomplete information is received, e.g., due to link or node failures. Such failures, when involving correct nodes, have the effect of degrading the number of nodes that corroborate other nodes' legitimate claims. We have taken into account message losses in our simulation study of the protocol (Sec. VII). In this section, instead, we evaluate the NPV resilience level considering only the behavior of nodes participating in the whole message exchange, that is, for which the verifier has collected all the required information.

\section{A. Faking own position: Independent adversaries}

We start by analyzing the attacks of the first type that can be launched by a single independent adversary in diverse network conditions, and explain the NPV protocol reactions they trigger. The discussion on the effects of the presence of multiple independent adversaries follows.

1) Basic attack: In the simplest scenario, a verifier $S$ runs the NPV protocol in presence of an adversary $M$, with which it shares no common neighbor. Let $p_{M}^{\prime}$ be the fake position that $M$ advertises: as briefly mentioned above, $M$ can announce a fake timing $t_{S M}^{\prime}$ in its REPLY, and a fake timing $t_{M}^{\prime}$ in its REPORT, so that $p_{M}^{\prime}$ is accepted by the verifier (i.e., $M \in \mathbb{V}_{S}$ ).

More precisely, the DST run by $S$ on $M$ verifies that the reciprocal distances are consistent, i.e., that $\left|d_{S M}-d_{M S}\right| \leq$ $2 \epsilon_{r}+\epsilon_{m}$, or:

$$
\left|\left(t_{S M}^{\prime}-t_{S}\right) \cdot c-\left(t_{M S}-t_{M}^{\prime}\right) \cdot c\right| \leq 2 \epsilon_{r}+\epsilon_{m}
$$

and that positions are also coherent with the distances, which implies ||$\left|p_{S}-p_{M}^{\prime} \|-d_{S M}\right| \leq 2 \epsilon_{p}+\epsilon_{r}$, or, equivalently:

$$
\left|\left\|p_{S}-p_{M}^{\prime}\right\|-\left(t_{S M}^{\prime}-t_{S}\right) \cdot c\right| \leq 2 \epsilon_{p}+\epsilon_{r}
$$

Therefore, the adversary must forge $t_{M}^{\prime}$ and $t_{S M}^{\prime}$, so that (1)-(2) still hold after its real position $p_{M}$ is replaced with $p_{M}^{\prime}$. Solving the equation system obtained by setting the error margin to zero in (1)-(2) and expressing the ToF using the node positions, we obtain:

$t_{M}^{\prime}=t_{M S}-\frac{\left\|p_{S}-p_{M}^{\prime}\right\|}{c}=t_{M}+\frac{\left\|p_{S}-p_{M}\right\|}{c}-\frac{\left\|p_{S}-p_{M}^{\prime}\right\|}{c}$

$t_{S M}^{\prime}=t_{S}+\frac{\left\|p_{S}-p_{M}^{\prime}\right\|}{c}=t_{S M}-\frac{\left\|p_{S}-p_{M}\right\|}{c}+\frac{\left\|p_{S}-p_{M}^{\prime}\right\|}{c}$

Note that $p_{M}^{\prime}$ is chosen by $M$, and that $M$ knows $t_{M}$ in (3) (since this is the actual transmission time of its own REPLY) and $t_{S M}$ in (4) (since this is the time at which it actually received $S$ 's POLL). Thus, we have a system of two equations in the two unknowns $t_{M}^{\prime}$ and $t_{S M}^{\prime} ; M$ can solve it if it knows $p_{S}$. We call this forging of transmission and reception timings with respect to $S$ the basic attack.

We stress that, in order to know $p_{S}, M$ must be a knowledgeable adversary, which implies two conditions: first, $M$ must have previously run the NPV protocol to discover the identity and position of its neighbors; second, the position of the verifier must have not changed since such discovery procedure. Clearly, as $M$ cannot foresee when $S$ starts the NPV protocol, such a condition is not easy to fulfill, especially in highly mobile environments. Nevertheless, if aware of $S$ 's location, $M$ could successfully run a basic attack, provided that the advertised position $p_{M}^{\prime}$ is within the proximity range $R$. As a consequence, the NPV marks isolated neighbors as unverifiable in the CST.

Let us now add to the previous scenario $n \geq 1$ nodes, $X_{1}, \ldots, X_{n}$, which are correct neighbors common to $S$ and $M$. The discussion above still holds, since the fake position advertised by $M$ must still pass the DST. Thus, $M$ has to know $S$ 's current position and to forge $t_{M}^{\prime}$ and $t_{S M}^{\prime}$ according to $p_{S}$ and $p_{M}^{\prime}$, as in (3)-(4). However, the presence of common neighbor(s) introduces two additional levels of security, which make the basic attack ineffectual.

First, the POLL and REPLY messages are anonymous; hence, upon their reception, even a knowledgeable $M$ does not know which node among $S, X_{1}, \ldots, X_{n}$ is the verifier. Nevertheless, in order to take part in the protocol, $M$ is forced to advertise the fake POLL reception time $t_{S M}^{\prime}$ in its REPLY, before receiving the REVEAL and discovering the identity of the verifier. The only option for $M$ is then to randomly guess who the verifier is and properly change $t_{S M}$ into $t_{S M}^{\prime}$, as in (4). This implies a probability of success in guessing the actual sender of the POLL equal to $1 /(n+1)$.

Second, in presence of shared (non-collinear) neighbors, $S$ can run the CST on the $\left(M, X_{i}\right)$ pairs, with $i=1, \ldots, n$. As the basic attack only forges messages transmission and reception timings with respect to $S$, the fake position $p_{M}^{\prime}$ will present discrepancies with the reciprocal reception times of REPLY messages at $M$ and $X_{i}$. This will result in a CST failure, revealing and thus preventing the attack.

2) REPLY-disregard attack: Whenever there are $n$ neighbors $X_{1}, \ldots, X_{n}$ common to $S$ and $M$, a possible strategy for the adversary, provided that it correctly guesses the identity of the verifier, is not to announce one or more of the common neighbors. That is, $M$ will not include the $\left(t_{X_{i} M}, i_{X_{i}}\right)$ data in its REPORT, thus deliberately denying to have received $X_{i}$ 's REPLY. We name this REPLY-disregard attack.

It follows that $S$ cannot perform a cross-check on the pairs $\left(M, X_{i}\right)$ in the CST. However, a REPLY-disregard attack does not bring any significant advantage to $M$. Indeed, the exclusion of (some or all of) the common neighbors reduces the system to one of the scenarios discussed for the basic attack, hence the adversary is at most tagged as unverifiable by $S$. More importantly, the maximum number of REPLYs an adversary can disregard is exactly one, otherwise it is classified as faulty in the MLT.

3) Hyperbola-based attack: This attack again attempts to fool the CST. More specifically, it scales up the basic attack by also forging the timings relative to the shared neighbor(s).

First, consider that $S$ and $M$ share a non-collinear common neighbor $X$. The CST on the $(M, X)$ pair at $S$ requires that $\left|d_{X M}-d_{M X}\right| \leq 2 \epsilon_{r}+\epsilon_{m}$ and ||$\left|p_{X}-p_{M} \|-d_{X M}\right| \leq 2 \epsilon_{p}+$ $\epsilon_{r}$. Applying the same substitutions as in (3) and (4), this 


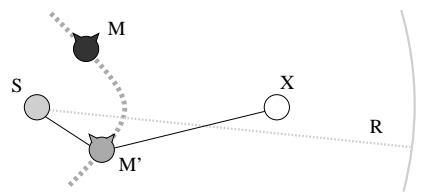

(a) One shared neighbor

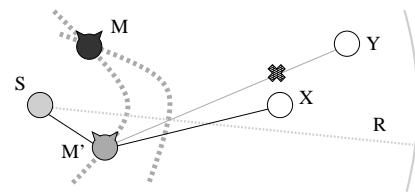

(b) Two shared neighbors
Fig. 3. Hyperbola-based attack: (a) If it correctly guesses $S$ 's identity, a knowledgeable adversary $M$ can forge timings with respect to $S$ and $X$ (black lines), so that they agree with any fake position $M^{\prime}$ lying on the hyperbola with foci in $S, X$, and passing by $M . M$ is unverifiable. (b) $M$ can only forge timings in agreement with fake positions that lie on both hyperbolae of foci in $S, X$, and $S, Y$, and passing by $M$. The only such point, i.e., the intersection, matches $M$ 's actual position. When moving on the hyperbola of foci $S$ and $X$, the timing with respect to Y (crossed gray line) is not verified.

means that $M$ is forced to advertise the following fake timings:

$$
\begin{aligned}
t_{M}^{\prime} & =t_{M}+\frac{\left\|p_{X}-p_{M}\right\|}{c}-\frac{\left\|p_{X}-p_{M}^{\prime}\right\|}{c} \\
t_{X M}^{\prime} & =t_{X M}-\frac{\left\|p_{X}-p_{M}\right\|}{c}+\frac{\left\|p_{X}-p_{M}^{\prime}\right\|}{c}
\end{aligned}
$$

If $M$ is knowledgeable, and thus aware of $X$ 's current position $p_{X}$, it can solve (6) and announce the forged $t_{X M}^{\prime}$ in its REPORT to $S$. However, (5) introduces a second expression for $t_{M}^{\prime}$, while $M$ can advertise only one $t_{M}^{\prime}$ in its REPORT. In order to pass both the DST and the CST, $M$ needs to announce a $t_{M}^{\prime}$ that satisfies (3) and (5), which implies:

$$
\left\|p_{S}-p_{M}\right\|-\left\|p_{S}-p_{M}^{\prime}\right\|=\left\|p_{X}-p_{M}\right\|-\left\|p_{X}-p_{M}^{\prime}\right\|
$$

In other words, $M$ is constrained to choose locations with the same distance increment (or decrement) from $S$ and $X$. In (7), $p_{S}, p_{X}$, and $p_{M}$ are fixed and known, hence the distances between $p_{S}$ and $p_{M}$, and between $p_{X}$ and $p_{M}$, can be considered as constant. We thus rewrite (7) as $\left\|p_{X}-p_{M}^{\prime}\right\|-\left\|p_{S}-p_{M}^{\prime}\right\|=k$. This is the equation, with the unknown $p_{M}^{\prime}$, of a hyperbola with foci in $p_{S}$ and $p_{X}$ that passes through $p_{M}$. It follows that only positions $p_{M}^{\prime}$ on such hyperbola can satisfy the four constraints in (3), (4), (5), and (6). As a conclusion, the hyperbola-based attack consists in advertising a fake position that lies on the aforementioned curve, as well as message transmission and reception times that validate such a position. An example is provided in Fig. 3(a).

Note that, in order to successfully perform a hyperbolabased attack, an adversary has to (i) know the position of both $S$ and $X$, (ii) correctly guess the identity of the verifier, and (iii) advertise a fake position only along a specific curve. Although these are restrictive conditions, the CST still marks as unverifiable the nodes that passed the DST but share only one neighbor with the verifier, so as to avoid any possibility of successful hyperbola-based attack.

We now consider a second correct, non-collinear node $Y \in \mathbb{N}_{S} \cap \mathbb{N}_{M}$, and show that, in such a scenario, also the hyperbola-based attack becomes futile. Note that no assumption is made on the connectivity between the two neighbors $X$ and $Y$. By extending the previous reasoning, in presence of two common correct neighbors, $X$ and $Y, M$ has to forge four time values, i.e., $t_{M}^{\prime}, t_{S M}^{\prime}, t_{X M}^{\prime}$, and $t_{Y M}^{\prime}$, so that six equations are satisfied, i.e., (3), (4), (5), (6) and two additional equations ${ }^{5}$ corresponding to the cross-check with the second common neighbor $Y$. This implies that the fake REPLY transmission time $t_{M}^{\prime}$ announced by $M$ must now fulfill three constraints, or, equivalently, $M$ must advertise a position $p_{M}^{\prime}$ that is equally farther from (or closer to) $S, X$ and $Y$ with respect to its actual location $p_{M}$. The only point satisfying such a condition lies at the intersection of three hyperbolae with foci in $p_{S}$ and $p_{X}, p_{S}$ and $p_{Y}, p_{X}$ and $p_{Y}$, respectively, and it corresponds to the real position of the adversary, $p_{M}$. In other words, if it shares two neighbors with $S$, an adversary cannot successfully claim to be at any location other than its actual one, not even if it is knowledgeable, it correctly guesses the role of all other nodes, and it performs a hyperbola-based attack. An example is provided in Fig. 3(b). We also stress that the presence of additional shared neighbors simply introduces other constraints on $t_{M}^{\prime}$, and thus further binds $M$ to its actual position.

Similarly, combining a hyperbola-based attack with a REPLY-disregard attack yields no chance of success. As a matter of fact, ignoring REPLY messages from one or multiple shared neighbors results in reverting the system to one of the cases previously analyzed, with the adversary being tagged at best as unverifiable.

4) Collinear attack: The above discussion shows that the presence of two or more correct common neighbors, which can be used to perform the cross-checks in the CST, is a condition that foils all the attack strategies introduced so far. There exists however a last type of attack, which we name collinear attack, that we need to discuss.

The collinear attack builds on the following geometrical property: if three points are collinear (i.e., lie on the same line), the hyperbola having as foci two of the points (one of which is that in the middle) and passing by the third degenerates into the half-line originating at the intermediate point and passing by the third one. This property implies that, if two shared neighbors $X$ and $Y$ lie between $S$ and the adversary $M$, and all four nodes are collinear, the hyperbolae that pin the actual position of the adversary $p_{M}$ degenerate to partially overlapping half-lines. This allows $M$ to forge timings relative to $S, X$ and $Y$ consistent with any fake position over the segment originating at the shared collinear neighbor that is closer to $M$, passing by $p_{M}$ and bounded by $R$. An example is given in Fig. 4(a).

In the more general case of any number of common neighbors to $S$ and $M$, the collinear attack would allow an adversary to appear correct to the shared neighbors that are collinear with it and $S$, as in Fig. 4(b). Again, this requires the adversary to be knowledgeable, to correctly guess the origin of POLL and REPLY messages it receives, and to limit the choice of its fake position to a specific segment.

As a countermeasure to collinear attacks, in the CST $S$ discards pairs of neighbors that announce collinear positions with it (Alg. 4, line 7). When collinear neighbors are dropped, a collinear attack results, for instance, in the adversary being tagged as unverifiable in Fig. 4(a) (since there are no non-

\footnotetext{
${ }^{5}$ The latter two equations can be obtained from (5)-(6) by replacing $p_{X}$, $t_{X M}$ and $t_{X M}^{\prime}$, respectively, with $p_{Y}, t_{Y M}$ and $t_{Y M}^{\prime}$.
} 


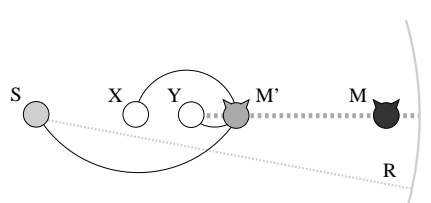

(a) Two shared neighbors

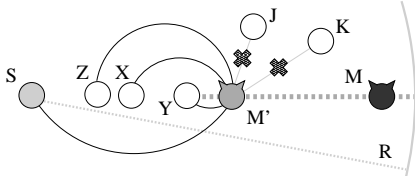

(b) Multiple shared neighbors
Fig. 4. Collinear attack: (a) The hyperbola of foci in $S, X(Y)$ and passing by $M$ degenerates into a half-line with origin in $X(Y)$. The hyperbolae intersection region, over which $M$ can announce a fake position $M^{\prime}$ with correct timings, becomes the segment originating at $Y$ and bounded by $R$ (dashed gray line). $M$ is unverifiable. (b) $M$ can announce timings that are consistent with its fake position $M^{\prime}$ with respect to the three collinear neighbors $X, Y$, and $Z$ (black lines). This is not possible with respect to $J$ and $K$ (crossed-out gray lines). As a countermeasure, collinear neighbors are not cross-checked in the CST. $M$ is tagged as faulty.

collinear shared neighbors) and as faulty in Fig. 4(b) (since there are two non-collinear shared neighbors).

We remark that, on the one hand, not allowing cross-checks that involve collinear nodes prevents collinear attacks. On the other, it reduces the number of correct neighbors that can contribute to identifying adversarial nodes. However, as shown in Sec. VII, this approach ensures high resilience to attacks as well as reliability in identifying correct nodes as verified.

5) Multiple independent adversaries: Multiple independent adversaries in the neighborhood of the verifier just damage each other, by announcing false positions that reciprocally spoil the time computations discussed in the previous sections. Thus, all cross-checks on pairs of non-colluding adversaries result in mismatches in the CST, increasing their chances to be tagged as faulty by the verifier.

Where multiple independent adversaries can harm the system is in the verification of correct neighbors. As a matter of fact, a node is tagged as verified if it passes the strict majority of cross-checks it undergoes. A correct node surrounded by several adversaries can thus be marked as faulty (unverifiable), if it shares with the verifier a number of non-collinear independent adversaries greater than (equal to) the number of noncollinear correct nodes. However, situations where a correct node shares mostly uncoordinated adversarial neighbors with the verifier are unlikely to occur in realistic scenarios, as also shown by our performance evaluation.

6) SUMMARY: We conclude that a single independent adversary cannot perform any successful attack against the NPV scheme. Indeed, in presence of a limited number of non-collinear neighbors in common with the verifier, a knowledgeable adversary can attempt one of the strategies outlined before, but it is tagged at most as unverifiable. When the shared neighborhood increases in size, the probability that the adversary is tagged as faulty rapidly grows to 1 . Multiple independent adversaries can only harm each other, thus reducing their probability of successfully announcing a fake position.

\section{B. Faking own position: Colluding adversaries}

We assume that colluders share out-of-band links with negligible latency, through which they exchange information, and can perform complex distributed computations. This notwithstanding, in the following we show that our scheme is

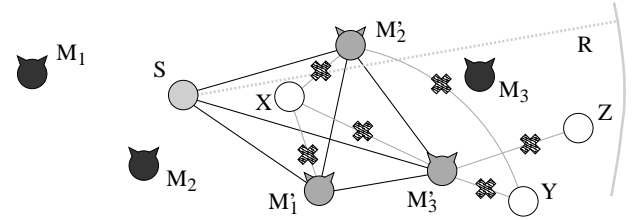

Fig. 5. Cooperative basic attack. The colluding adversaries $M_{1}, M_{2}$, and $M_{3}$ can forge timings that validate their fake positions $M_{1}^{\prime}, M_{2}^{\prime}$, and $M_{3}^{\prime}$ with respect to $S$, as well as to each other (black lines). However, cross-checks with non-colluding neighbors fail (crossed-out gray lines). $M_{1}$, sharing with $S$ the other two colluders and $X$, is tagged as verified. $M_{2}$, sharing with $S$ the other two colluders and $X, Y$, is unverifiable. $M_{3}$, sharing with $S$ the other two colluders and $X, Y, Z$, is marked as faulty.

resistant to coordinated attacks ${ }^{6}$ as well, unless the presence of colluding adversaries in the neighborhood of the verifier becomes overwhelming.

1) Basic attack: The simplest way adversarial nodes can cooperate to make the verifier $S$ trust the fake positions they announce is by extending the basic attack introduced in Sec. VI-A1. More precisely, other than individually announcing POLL reception timings that agree with their fake positions, colluding adversaries can mutually validate the false information they generate. They can forge the reception times of reciprocal REPLY messages, so that all cross-checks in the CST involving the colluders are passed. A perfect cooperation thus results in the colluding adversaries ability to alter all distances between them without being noticed.

We remark that the adversaries still need to know $S$ 's position in order to compute and advertise timings that confirm their fake position. This time, however, if at least three adversaries cooperate to perform the attack, they do not need to be knowledgeable. As a matter of fact, they can exploit their real positions and POLL reception times to multilaterate the coordinates of the verifier.

Our NPV correctly identifies such a basic attack through the CST, as long as the majority of the (non-collinear) neighbors shared by $S$ and an adversary are not colluding with the latter. An example is shown in Fig. 5.

2) REPLY-disregard attack: As in the case of independent adversaries, multiple colluders can gain advantage by ignoring REPLY messages from non-colluding nodes. This lets them avoid cross-checks that could result in mismatches, and, eventually, in being tagged as faulty. Indeed, $n \geq 3$ colluders could disregard the REPLY received from all non-colluding nodes and still advertise a number $n-1 \geq 2$ of neighbors that would report consistent timings with theirs - a sufficient condition to pass the CST. This behavior, however, is properly handled in our NPV by the MLT, which tags as faulty a neighbor that disregarded (intentionally or not) two or more REPLY messages and announced a location other than the multilaterated one.

3) Hyperbola-based attack: The colluding attackers agree not only on the position of the verifier (either guessed or multilaterated), but also pick a non-collinear common neighbor, $X$, that they share with $S$ : each colluder then computes the hyperbola with foci $S, X$, and passing through its own real position, and announces a fake location on such a curve.

\footnotetext{
${ }^{6}$ Note that, since our NPV exploits ToF-based ranging, wormhole attacks reduce to Sybil attacks, as discussed in Sec. VI-D.
} 


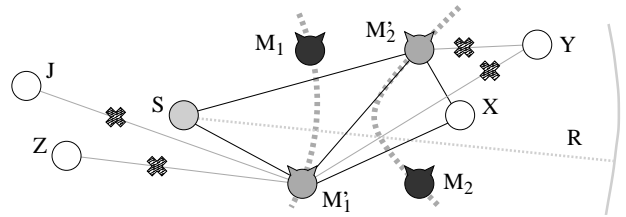

Fig. 6. Cooperative hyperbola-based attack: If two colluding adversaries, $M_{1}$ and $M_{2}$, are knowledgeable and correctly guess the identity of $S$, they can forge timings with respect to $S$ and $X$ as well as to each other (black lines) and announce the fake positions $M_{1}^{\prime}$ and $M_{2}^{\prime}$, respectively. $M_{1}^{\prime}\left(M_{2}^{\prime}\right)$ can be any point on the hyperbola with foci in $S, X$, and passing by $M_{1}\left(M_{2}\right)$. However, $M_{1}$ shares 4 correct and one colluding, non-collinear neighbors with $S$, while $M_{2}$ shares 2 correct and one colluding, non-collinear neighbors with $S$, hence $M_{1}$ is tagged as faulty and $M_{2}$ is tagged as verified.

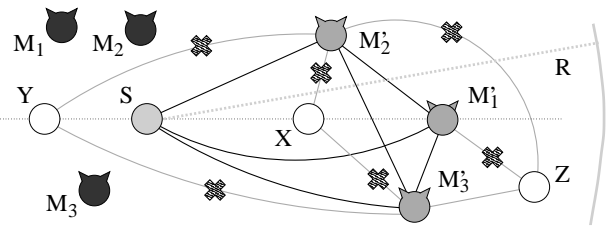

Fig. 7. Cooperative collinear attack. The adversary $M_{1}$ announces a fake position $M_{1}^{\prime}$ that is collinear with the verifier $S$ and the non-colluding neighbors $X, Y$, avoiding cross-checks with the latter two. $M_{2}$ and $M_{3}$, colluding with $M_{1}$, declare non-collinear false locations $\left(M_{2}^{\prime}, M_{3}^{\prime}\right)$, thus guaranteeing to $M_{1}$ a majority of validated cross-checks. The adversary $M_{1}$ is tagged as verified, while $M_{2}$ and $M_{3}$ are marked as faulty.

This allows the adversaries to announce correct links (i) with the verifier, (ii) among themselves, and (iii) with the selected neighbor $X$, which becomes an involuntary ally in the attack. Again, the location of $X$ must be randomly guessed by two colluders, while it can be multilaterated by three or more cooperating adversaries.

In presence of such a hyperbola-based attack, the CST correctly tags an adversary $M$ as faulty if the non-collinear common neighbors between the verifier and $M$ that do not collude with $M$ outnumber the colluding ones by 3 . Note that the two additional correct neighbors are required to counter the effect of $X$ unintentionally taking part into the attack. An example is depicted in Fig. 6.

4) Collinear attack: Unlike independent adversaries (Sec. VI-A4), multiple colluders can take advantage of a collinear attack. In particular, one or more adversaries can purposely announce positions that are collinear with those of some non-colluding neighbors, so as to avoid cross-checks with them. Then, they can rely on colluders that declared non-collinear positions to pass the CST, as in Fig. 7.

In other words, colluders can launch a collinear attack as a legal mean to avoid unwanted neighbors. Such a gain comes at the cost of a restricted freedom of movement, since the fake position must lie on a specific segment (see Sec. VI-A4). The robustness of our NPV to this kind of attacks depends on the network layout: environments where nodes tend to form straight topologies (such as vehicular ones) are more prone to suffer from collinear attacks. In general, the NPV is resistant to collinear attacks as long as the majority of the shared neighbors are not colluding or collinear.

5) SUMMARY: As a conclusion on coordinated attacks, it is the nature of the neighborhood that determines the performance of the NPV scheme in presence of colluders.
However, the simulation results in Sec. VII show that, in realistic environments, our solution is very robust even to attacks launched by large groups of knowledgeable colluders.

\section{Discrediting other neighbors}

A different objective of the adversary can be to discredit other nodes by inducing the verifier to tag them as faulty or unverifiable. To this end, an adversary $M$ needs to announce a fake timing $t_{X M}^{\prime}$ (i.e., the time at which $M$ claims it has received $X$ 's REPLY), for any neighbor $X \in \mathbb{N}_{S} \cap \mathbb{N}_{M}$ that it wants to discredit. By doing so, $M$ can disrupt the crosschecks made by $S$ on the pair $(X, M)$ in the CST. When launched by a single adversary, such an attack can succeed if there are only two additional correct nodes (which are tagged as unverifiable by $S$ ). In all other configurations, a single adversary cannot affect the assessment of other correct nodes.

When launched by multiple adversaries, no matter whether they are independent or colluding, the effect of this attack is the same as the one highlighted in Sec. VI-A5. We recall that our NPV protocol provides protection to a correct node $X$, as long as the number of adversarial neighbors it shares with the verifier $S$ is lower than that of correct common neighbors. Vice versa, if the number of adversarial shared neighbors trying to discredit $X$ is greater than (equal to) the number of correct common neighbors, $X$ is tagged as faulty (unverifiable).

\section{Other attacks}

Jamming. This is the only external attack that can harm the system. Any adversary (internal or external) can jam the channel and erase REPLY or REPORT messages. However, to succeed, $M$ should jam the medium continuously for a long time, since it cannot know when exactly a node will transmit its REPLY or REPORT. Or, $M$ could erase the REVEAL, but, again, jamming should cover the entire $T_{j i t t e r}$ time. Overall, there is no easy point to target: a jammer has to act throughout the NPV execution, which implies a high energy consumption and is a disruptive action possible against any wireless protocol. In addition, mobility makes it harder to repeatedly jam different instances of the NPV protocol run by the same verifier.

Clogging. An adversary could initiate the NPV protocol multiple times in a short period and get repeated REPLY and REPORT messages from other nodes, so as to congest the channel. In particular, REPORTs are larger in size, thus likely cause the most damage. However, NPV has a way of preventing that: the initiator must unveil its identity before such messages are transmitted by neighbors. An exceedingly frequent initiator can be identified, and its REVEALs ignored, thanks to the use of certified keys. REPLYs instead are small in size and are broadcast messages (thus require no ACK): their damage is limited, but their unnecessary transmission is much harder to thwart. Indeed, REPLY messages are sent after an anonymous POLL; such an anonymity is a hard-to dismiss requirement, since it is instrumental for keeping the identity of the verifier hidden. As a general rule, correct nodes can reasonably self-limit their responses if POLLs arrive at excessive rates. 
Adversarial use of directional antennas. Assume that adversaries are equipped with directional antennas and multiple radio interfaces. As a correct node $S$ starts the NPV protocol, a knowledgeable adversary $M$ can send different REPLYs through each interface at different time instants: any correct neighbor $X$ would record a time $t_{M X}^{\prime}$, compliant with the fake position $p_{M}^{\prime}$, allowing $M$ to pass the cross-check with $X$ in the CST. If $M$ can fool a sufficient number of neighbors, it is tagged as verified. However, $M$ needs as many directional antennas and radio interfaces as the number of neighbors it wants to fool, hoping that no two such neighbors are within the beam of the same antenna. The complexity, cost, and chances of failure make this attack hardly viable. We also remark that, since our approach exploits ToF-based ranging, such an attack cannot be launched by using a steerable antenna, which takes an exceedingly long time to swap from one sector to another. Sybil and relay (wormhole) attacks. An adversary can assume several trusted identities, $\mathcal{M}=\left\{M_{1}, \ldots, M_{I}\right\}$, if (i) it owns several certificated pairs of public/private keys (Sybil attack), or (ii) it impersonates colluding adversaries at the end of wormholes. The availability of several identities could be used by an adversary to acquire its neighbor positions, i.e., to become knowledgeable. However, as shown in Sec. VI-A, attacks launched by independent, knowledgeable adversaries have no chance of success. Furthermore, by announcing timings that are consistent among the identities in $\mathcal{M}$, the adversary can behave as a group of colluders of size $I$. The analysis in Sec. VI-B thus applies to such attacks as well, except for the fact that the adversary cannot acquire the position of other nodes through triangulation. A verifier suspecting ${ }^{7}$ that this attack is being launched can run the MLT to determine whether messages from nodes in $\mathcal{M}$ come from the same location.

\section{ViI. Performance evaluation}

We evaluated the performance of our NPV protocol in a vehicular scenario. Results obtained in a pedestrian scenario are available as Supplemental Material.

We focus on knowledgeable adversaries whose goal is to make the verifier believe their fake positions, and we describe the best attack strategy they can adopt in Sec. VII-A. Such a strategy, which depends on the neighborhood of the adversary and builds on a combination of the attacks described in Secs. VI-A and VI-B, will be assumed while deriving the results shown in Sec. VII-B.

The results, which therefore represent a worst-case analysis of the proposed NPV, are shown in terms of the probability that the tests return false positives and false negatives as well as of the probability that a (correct or adversary) node is tagged as unverifiable. In addition, we plot the average difference between the true position of a successful adversary and the fake position it advertises, as well as the overhead introduced by our NPV scheme. The results on attacks aimed at discrediting the position of other nodes are omitted, since they are very close to those we present later in this section.

\footnotetext{
${ }^{7}$ An example of suspect situation is the case where the neighborhood of the verifier is split into groups of nodes, whose members pass the cross-checks in the CST only with the nodes belonging to the same group.
}

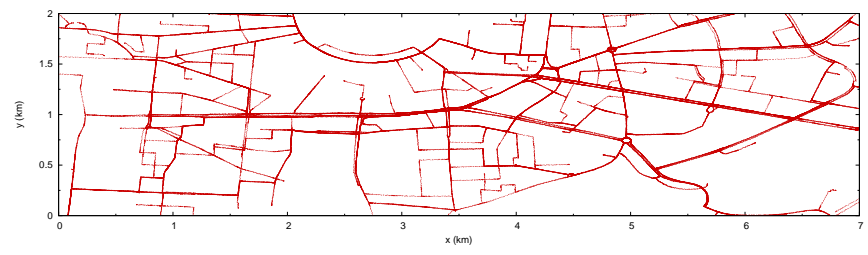

Fig. 8. Road layout of the $7 \times 3 \mathrm{~km}^{2}$ vehicular scenario.

\section{A. Adversaries attack strategy}

The adversary decision on the kind of attack to launch is driven by the tradeoff between the chances of success and the freedom of choice on its fake position. The basic attack allows the adversary to choose any false position, but it requires a high percentage of colluders in the neighborhood in order to be successful. The hyperbola-based attack implies less freedom of choice but has higher chances of success. The collinear attack pins the adversary into a precise angle with the verifier, and strictly bounds its distance from the verifier itself. However, if the network topology features a sufficient number of collinear nodes, this attack has the highest success probability.

It follows from Sec. VI that the best strategy that an adversary can adopt depends on its neighborhood. Firstly, if it colludes with other adversaries outnumbering the noncolluding neighbors, a basic attack is launched. Otherwise, if the ratio between colluding and non-colluding neighbors is not greater than (but close enough to) 1, a hyperbola-based attack is attempted. As a third option, if non-colluding neighbors greatly outnumber the colluding ones, but some of the former are collinear with the verifier and among themselves, the adversary launches a collinear attack. Through it, the adversary can have the non-colluding, collinear neighbors thrown out of the cross-checks in the CST. If none of the above conditions are met, the adversary picks a hyperbola-based attack, i.e., the one with the highest chances of success in absence of noncolluding, collinear neighbors. Also, an adversary always runs a REPLY-disregard on one non-colluding neighbor, avoiding a mismatch with it. Recall that disregarding just one REPLY does not trigger the MLT on the adversary.

\section{B. Results}

We employed movement traces representing vehicle traffic over a real-world road topology. More precisely, we considered car movements within a $20 \mathrm{~km}^{2}$ portion of the Karlsruhe urban area depicted in Fig. 8, extracting 3 hours of vehicular mobility that reproduce mild to heavy traffic density conditions. These synthetic traces were generated using the IDM-LC model of the VanetMobiSim simulator, which takes into account car-tocar interactions, traffic lights, stop signs and lane changes, and has been proven to realistically reproduce vehicular movement patters in urban scenarios [31].

In our simulations, we set $T_{\max }=200 \mathrm{~ms}, T_{j i t t e r}=50 \mathrm{~ms}$, $\Delta=1 \mathrm{~ms}$ and assume that CSMA/CA is used to access the wireless medium, hence messages can be lost due to collisions. Unless otherwise specified, we fix the proximity range, $R$, which is equal to the maximum nominal transmission range, 


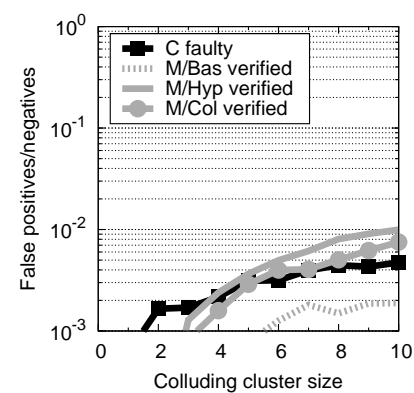

(a)

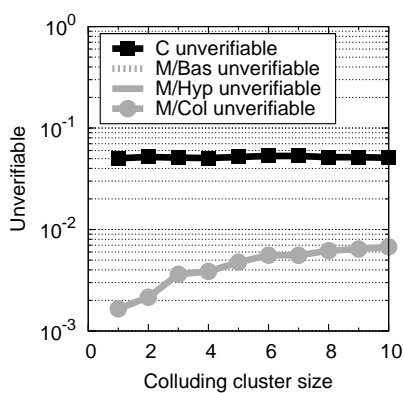

(b)

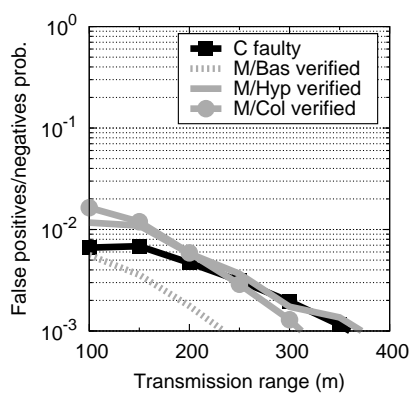

(c)

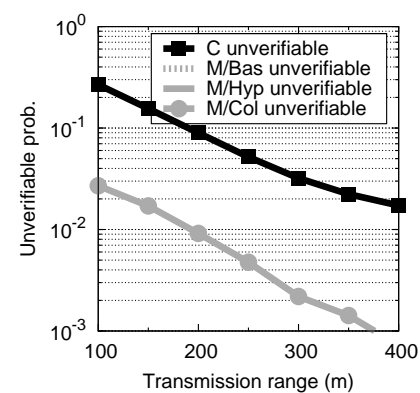

(d)

Fig. 9. Probability that a neighbor is tagged incorrectly or as unverifiable, versus the colluder cluster size (a,b), and versus $R$ (c,d). C: correct; M/Bas, M/Hyp and M/Col: adversaries launching the basic, hyperbola-based and collinear attack, each combined with the REPLY-disregard attack.

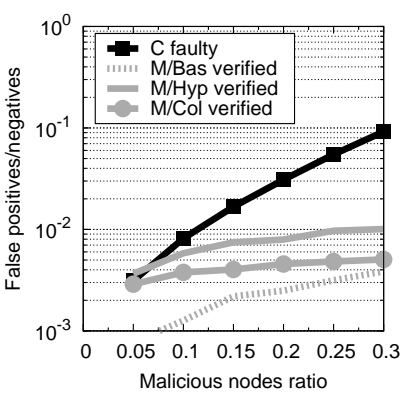

(a)

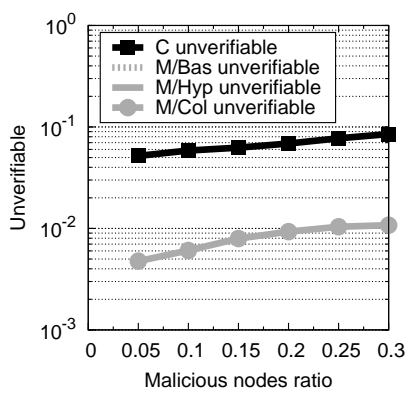

(b)

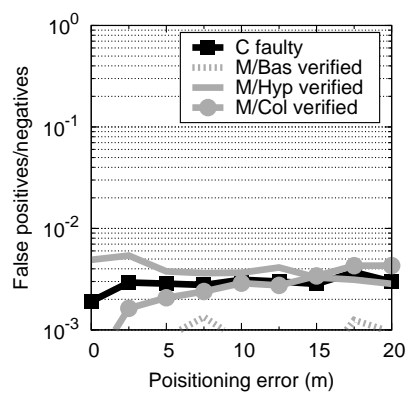

(c)

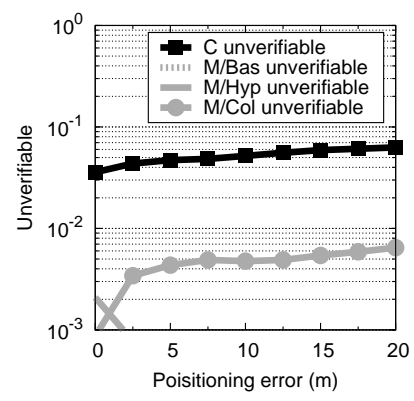

(d)

Fig. 10. Probability that a neighbor is tagged incorrectly or as unverifiable, versus the ratio of adversaries (a,b), and position error (c,d). C: correct; M/Bas, M/Hyp and M/Col: adversaries launching the basic, hyperbola-based and collinear attack, each combined with the REPLY-disregard attack.

to $250 \mathrm{~m}$ (resulting in an average neighborhood size of 73.4 nodes), while $\epsilon_{r}=6.8 \mathrm{~m}, \epsilon_{p}=10 \mathrm{~m}$, and the tolerance value $\epsilon_{m}=5 \mathrm{~m}$ (roughly corresponding to the case of two vehicles moving at $50 \mathrm{~km} / \mathrm{h}$ in opposite directions).

To evaluate the performance of our NPV, at every simulation second we randomly select $1 \%$ of the nodes as verifiers. Then, for each verifier, we compare the outcome of the verification tests with the actual nature of the neighbors. We consider colluding adversaries acting in groups, referred to as clusters. Note that a colluding cluster size equal to 1 corresponds to independent attacks. Also, adversaries are knowledgeable, i.e., they perfectly know the identity and location of all colluding and non-colluding neighbors, and always adopt the best attack strategy as described in Sec. VII-A. In the following, unless otherwise specified, adversaries amount to $5 \%$ of the overall nodes and are divided into clusters of 5 colluders each.

In the legend of the plots, $\mathbf{C}$ stands for correct node (e.g., the label "C faulty" refers to the probability of false positives), while $\mathbf{M} / \mathbf{B a s}, \mathbf{M} / \mathbf{H y p}$ and $\mathbf{M} / \mathbf{C o l}$ stand for adversaries launching, respectively, the basic, hyperbola-based and collinear attack (e.g., the label "M/Bas verified" refers to the probability of false negatives due to basic attacks).

We first examine the NPV protocol performance for different values of colluding cluster sizes and $R=250 \mathrm{~m}$ (Figs. 9(a) and $9(\mathrm{~b})$ ).

The false negative/positive probability in Fig. 9(a) clearly shows that (i) the chance of wrong classification reaches 0.01 only for a very large adversarial cluster size, namely 10, (ii) the hyperbola-based and the collinear attacks are the most threatening and (iii) an attack by the colluders is most effective in passing themselves off as verified when there are at least three of them. The cluster size also affects the colluders ability to disrupt the positioning of correct nodes, which exhibit as high as a $0.4 \%$ chance to be tagged as faulty.

Conversely, as shown in Fig. 9(b), the cluster size does not cause more correct nodes to be unverifiable, since the main reason for correct nodes to be tagged as unverifiable is the lack of non-collinear neighbors that can verify them. The chance for an adversary to be unverifiable increases with the cluster size, although it is significant only in case of collinear attacks. This is in agreement with the fact that the outcome of the collinear attack is the avoidance of a sizable number of cross-checks between the adversary and correct nodes, thus likely leading the adversary to be tagged as unverifiable.

The neighborhood size proves to play an important role, as evident in Figs. 9(c) and 9(d) where we consider a 5-colluder cluster and vary the transmission range. A small $R$ (hence few neighbors) affects the NPV capability to correctly tag a node. Widening the transmission range with a fixed colluding cluster size significantly favors the verifier, allowing it to reach a conclusive and exact verdict on either correct or adversary nodes: the larger the $R$, the higher the number of cross-checks involving correct nodes in the CST. We note that, for transmission ranges larger than $300 \mathrm{~m}$, we obtain false positive/negative probabilities that are smaller than 0.001 . Below $150-\mathrm{m}$ ranges (corresponding to an average neighborhood size of 12 nodes), such probabilities are still 0.01 .

Beside the impact of the cluster size and of the transmission range, it is important to understand the effect of the percentage of adversaries in the vehicular network. Thus, in Fig. 10(a) 


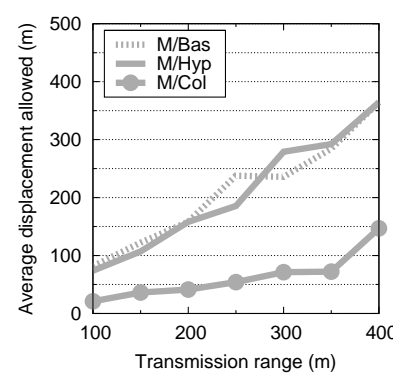

(a)

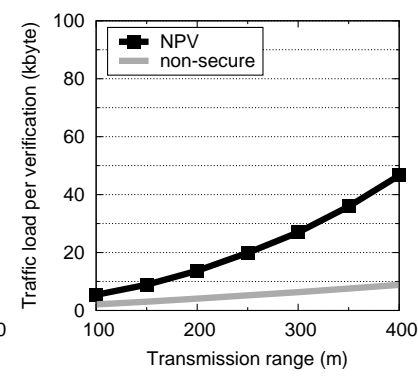

(b)
Fig. 11. Displacement gain of adversaries running a successful attack against the NPV (a) and traffic load induced by one instance of the protocol (b).

we fix $R$ to $250 \mathrm{~m}$ and the cluster size to 5 , and we show the robustness of our NPV to the density of adversaries: the probability that adversaries are verified increases ever so slightly with their density. The highest effect is on the probability of correct nodes being tagged as faulty, which however reaches its highest value (0.1) only for $30 \%$ of adversaries in the network. A further effect of the growing presence of adversaries, as shown in Fig. 10(b), is the unverifiable tag being slapped onto more correct nodes. A final observation can be made looking at the false positive/negative probability as the positioning error varies (Figs. 10(c) and 10(d)). Interestingly, for any positioning error different from 0 , the metrics are only marginally affected.

Finally, we further increase the level of detail of our analysis and study the advantage obtained by adversaries that perform a successful attack against the NPV protocol. Such an adversarial gain is expressed in terms of spatial displacement, i.e., difference of position between the real and fraudulently advertised locations of the successful attacker: clearly, a larger displacement range implies a higher freedom of movement, which, in turn, enables potentially more dangerous actions against the system. The results in Fig. 11(a) are broken down based on the type of attack launched by the successful adversary, and are limited to the impact of the transmission range, since the other parameters did not show significant influence on the displacement of successful attackers.

We can observe that successful collinear attacks yield small advantage for adversaries, who are forced to announce positions quite close to their real locations. Moreover, we recall that these attacks constrain adversaries to advertise fake positions along a precise axis, thus further limiting their freedom of movement. We can conclude that collinear attacks, typically those with the highest chances of success as previously discussed, are also those resulting in the smallest gain for the adversaries. Conversely, basic attacks allow the largest average displacements, but we showed that they have extremely low success probability. The hyperbola-based attacks appear then to be the most dangerous ones, if the displacement gain is taken into consideration. However, such a gain becomes significant only for large transmission ranges, in presence of which we already observed that the actual success probability of the attacks becomes negligible.

Finally, we comment on the overhead introduced by our scheme. The NPV protocol generates at most $2 n+2$ messages for one execution initiated by a verifier with $n$ communication neighbors. Also, NPV messages are relatively small in size: with SHA-1 hashing and ECDSA-160 encryption [27], the length of signatures is 21 bytes (with coordinates compression). Assuming that messages include headers with 4-byte source and destination identifiers and 1-byte message type field, POLL, REPLY, and REVEAL are 26, 71, and 67 bytes in size, respectively. The REPORT length depends on the quantity of common neighbor data it carries, amounting to 4 bytes per shared neighbor: information on more than 360 neighbors can thus fit in a single IP packet.

Fig. 11(b) portrays the traffic induced on the network by one instance of the NPV protocol. The plot only accounts for transmission range variations since, once more, the other parameters do not have an impact on the overhead. We can observe that security comes at a cost, since the traffic load of the NPV protocol is higher than that of a basic non-secure neighbor position discovery, consisting of only one poll and associated position replies from neighbors. More precisely, the NPV protocol overhead is comparable to that of the nonsecure discovery for smaller transmission ranges, while the difference tends to increase for larger ranges. However, the cost of the NPV protocol is affordable in absolute terms, since one run requires just a few tens of kbytes to be exchanged among nodes, even in presence of dense networks and large transmission ranges. Note that the results above do not take into account the overhead induced by the distribution of certificates, as it is out of the scope of this work (the interested reader can refer to [26]).

Summary. Given that we assumed the best possible conditions for the adversaries, the above results prove our NPV to be highly resilient to attacks. Indeed, we observed typical probabilities of false positives/negatives below $1 \%$, while that of a node being tagged as unverifiable is below 5\%. Moreover, we showed that a significant portion of the successful attacks yields small advantage to the adversaries in terms of displacement. Finally, the overhead introduced by the NPV protocol is reasonable, as it does not exceed a few tens of kbytes even in the most critical conditions.

\section{CONCLUSION}

We presented a distributed solution for NPV, which allows any node in a mobile ad hoc network to verify the position of its communication neighbors without relying on a-priori trustworthy nodes. Our analysis showed that our protocol is very robust to attacks by independent as well as colluding adversaries, even when they have perfect knowledge of the neighborhood of the verifier. Simulation results confirm that our solution is effective in identifying nodes advertising false positions, while keeping the probability of false positives low. Only an overwhelming presence of colluding adversaries in the neighborhood of the verifier, or the unlikely presence of fullycollinear network topologies, can degrade the effectiveness of our NPV. Future work will aim at integrating the NPV protocol in higher-layer protocols, as well as at extending it to a proactive paradigm, useful in presence of applications that need each node to constantly verify the position of its neighbors. 


\section{REFERENCES}

[1] 1609.2-2006: IEEE Trial-Use Standard for Wireless Access in Vehicular Environments - Security Services for Applications and Management Messages, 2006.

[2] P. Papadimitratos, L. Buttyan, T. Holczer, E. Schoch, J. Freudiger, M. Raya, Z. Ma, F. Kargl, A. Kung, J.-P. Hubaux, "Secure Vehicular Communications: Design and Architecture," IEEE Comm. Mag., vol. 46, no. 11, pp. 100-109, Nov. 2008.

[3] P. Papadimitratos, A. Jovanovic, "GNSS-based Positioning: Attacks and Countermeasures," IEEE Milcom, San Diego, CA, Nov. 2008

[4] L. Lazos, R. Poovendran, "HiRLoc: High-resolution Robust Localization for Wireless Sensor Networks," IEEE JSAC, vol. 24, no. 2, pp. 233-246, Feb. 2006.

[5] R. Poovendran, L. Lazos, "A Graph Theoretic Framework for Preventing the Wormhole Attack," Wireless Networks, 2007.

[6] S. Zhong, M. Jadliwala, S. Upadhyaya, C. Qiao, "Towards a Theory of Robust Localization Against Malicious Beacon Nodes," IEEE Infocom, Phoenix, AZ, Apr. 2008.

[7] P. Papadimitratos, M. Poturalski, P. Schaller, P. Lafourcade, D. Basin, S Čapkun, J.-P. Hubaux, "Secure Neighborhood Discovery: A Fundamental Element for Mobile Ad Hoc Networks," IEEE Comm. Mag., vol. 46, no. 2, Feb. 2008.

[8] Y.-C. Hu, A. Perrig, D. B. Johnson, "Packet Leashes: A Defense against Wormhole Attacks in Wireless Networks," IEEE Infocom, S. Francisco, CA, Apr. 2003.

[9] J. Eriksson, S. Krishnamurthy, M. Faloutsos, "TrueLink: A Practical Countermeasure to the Wormhole Attack in Wireless Networks," IEEE ICNP, Santa Barbara, CA, Nov. 2006.

[10] R. Maheshwari, J. Gao, S. Das, "Detecting Wormhole Attacks in Wireless Networks Using Connectivity Information," IEEE Infocom, Anchorage, AK, Apr. 2007

[11] R. Shokri, M. Poturalski, G. Ravot, P. Papadimitratos, J.-P. Hubaux, "A Practical Secure Neighbor Verification Protocol for Wireless Sensor Networks," ACM WiSec, Zurich, Switzerland, Mar. 2009.

[12] M. Poturalski, P. Papadimitratos, J.-P. Hubaux, "Secure Neighbor Discovery in Wireless Networks: Formal Investigation of Possibility," ACM ASIACCS, Tokyo, Japan, Mar. 2008.

[13] M. Poturalksi, P. Papadimitratos, J.-P. Hubaux, "Towards Provable Secure Neighbor Discovery in Wireless Networks," Workshop on Formal Methods in Security Engineering, Alexandria, VA, Oct. 2008.

[14] E. Ekici, S. Vural, J. McNair, D. Al-Abri, "Secure Probabilistic Location Verification in Randomly Deployed Wireless Sensor Networks," Elsevier Ad Hoc Networks, vol. 6, no. 2, pp. 195-209, 2008.

[15] J. Chiang, J. Haas, Y. Hu, "Secure and Precise Location Verification Using Distance Bounding and Simultaneous Multilateration," ACM WiSec, Zurich, Switzerland, Mar. 2009.

[16] S. Čapkun, K. Rasmussen, M. Cagalj, M. Srivastava, "Secure Location Verification with Hidden and Mobile Base Stations," IEEE Trans. on Mobile Computing, vol. 7, no. 4, pp.470-483, 2008.

[17] S. Čapkun, J.-P. Hubaux, "Secure Positioning in Wireless Networks," IEEE JSAC, vol. 24, no. 2, pp. 221-232, 2006.

[18] A. Vora, M. Nesterenko, "Secure Location Verification Using Radio Broadcast," IEEE Trans. on Dependable and Secure Computing, vol. 3, no. 4, pp. 377-385, 2006.

[19] J. Hwang, T. He, Y. Kim, "Detecting Phantom Nodes in Wireless Sensor Networks," IEEE Infocom, Anchorage, AK, May 2007.

[20] T. Leinmüller, C. Maihöfer, E. Schoch, F. Kargl, "Improved Security in Geographic Ad Hoc Routing through Autonomous Position Verification," ACM VANET, Los Angeles, CA, Sept. 2006.

[21] J.-H. Song, V. Wong, V. Leung, "Secure Location Verification for Vehicular Ad-Hoc Networks," IEEE Globecom, New Orleans, LO, Dec. 2008.

[22] M. Fiore, C. Casetti, C.-F. Chiasserini, P. Papadimitratos, "Secure Neighbor Position Discovery in Vehicular Networks," IEEE/IFIP MedHoc-Net, Favignana, Italy, June 2011

[23] Federal Highway Administration, "High Accuracy-Nationwide Differential Global Positioning System Test and Analysis: Phase II Report," FHWA-HRT-05-034, July 2005.

[24] http://www.nanotron.com/EN/pdf/Factsheet_nanoLOC-NA5TR1.pdf.

[25] PRECIOSA: Privacy Enabled Capability in Co-operative Systems and Safety Applications, http://www.preciosa-project.org.

[26] G. Calandriello, P. Papadimitratos, A. Lioy, J.-P. Hubaux, "On the Performance of Secure Vehicular Communication Systems," IEEE Trans. on Dependable and Secure Computing, vol. 8, no. 6, pp. 898-912, 2011.

[27] IEEE 1363a 2004. IEEE Standard Specifications for Public-key Cryptography-Amendment 1: Additional Techniques, 2004.
[28] M. Ciurana, F. Barcelo-Arroyo, F. Izquierdo, "A Ranging System with IEEE 802.11 Data Frames," IEEE Radio and Wireless Symposium, Jan. 2007.

[29] F. Carpenter, S. Srikanteswara, A. Brown, "Software Defined Radio Test Bed for Integrated Communications and Navigation Applications," Software Defined Radio Technical Conference, Nov. 2004.

[30] E. Del Re, L. S. Ronga, L. Vettori, L. Lo Presti, E. Falletti, M Pini, "Software Defined Radio Terminal for Assisted Localization in Emergency Situations," CTIF Wireless Vitae, May 2009.

[31] J. Härri, M. Fiore, F. Filali, C. Bonnet, "Vehicular Mobility Simulation with VanetMobiSim," Trans. Soc. Modeling \& Simulation, 2009.

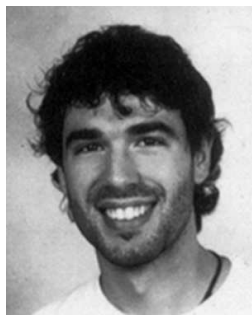

Marco Fiore (M'05) is an Assistant Professor at INSA Lyon, and an INRIA researcher within the SWING team hosted by the CITI Lab. He received his $\mathrm{PhD}$ from Politecnico di Torino, in 2008, and M.Sc degrees from University of Illinois at Chicago and Politecnico di Torino, in 2003 and 2004, respectively. He has been a visiting researcher at Rice University and Universitat Politecnica de Catalunya. His research interests are in the field of mobile networking, with focus on vehicular networks.

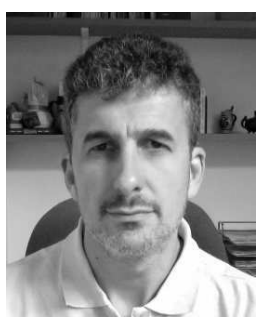

Claudio Casetti (M'05) graduated in Electrical Engineering from Politecnico di Torino in 1992 and received his $\mathrm{PhD}$ in Electronic Engineering from the same institution in 1997. He is an Assistant Professor at the Dipartimento di Elettronica of Politecnico di Torino. He has coauthored more than 130 journal and conference papers in the fields of networking and holds three patents. His interests focus on ad hoc wireless networks and vehicular networks.

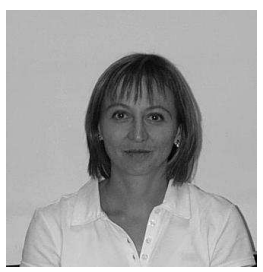

Carla-Fabiana Chiasserini (M'98 SM'09) received her Ph.D. from Politecnico di Torino in 2000. She has worked as a visiting researcher at UCSD in 1998-2003, and she is currently an Associate Professor at Politecnico di Torino. Her research interests include architectures, protocols, and performance analysis of wireless networks. Dr. Chiasserini has published over 190 papers in prestigious journals and leading international conferences, and she serves as Associated Editor of several journals.

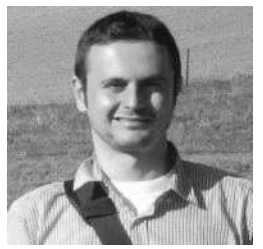

Panagiotis Papadimitratos (M'00) received his $\mathrm{PhD}$ from Cornell University, Ithaca, NY, USA. He was a post-doctoral fellow at Virginia Tech, Blacksburg, VA, a scientist at EPFL, Lausanne, Switzerland, and a visiting faculty member at Politecnico di Torino. He is now an Associate Professor in the School of Electrical Engineering at KTH, Stockholm, Sweden. His research is concerned with security and networked systems, with more than 70 related technical publications. 